\title{
CONTACT MECHANICS IN FRETTING FATIGUE
}

\author{
J. De Pauw ${ }^{1}$, P. De Baets ${ }^{1}$, W. De Waele ${ }^{1}$, R. Hojjati ${ }^{1}$ \\ ${ }^{1}$ Ghent University, Laboratory Soete, Belgium
}

\begin{abstract}
This paper studies the contact mechanics in a line contact during fretting fatigue conditions. In literature one can find numerical and analytical solutions of normal and tangential stresses for a variety of loading cases. However, a unified solution valid for all loading cases during fretting fatigue conditions is not available. We present in this paper a strategy to combine existing contact mechanics theories into a unified calculation procedure. Therefore, the relevant contact mechanics theories for an idealized cylinder-on-flat contact are selected and bundled. Two clear flowcharts group the existing theories, which results in a unified strategy that can easily be implemented in a programming language. A Matlab script was programmed and calculates the normal and tangential stress distribution based on the applied forces, the geometry of the contact, the coefficient of friction and the material properties. The present theory can be used to automate the calculation of the stress distributions, or as validation of new numerical techniques. The script is modular and can be extended to calculate the lifetime of a component, by adding lifetime criteria.
\end{abstract}

Keywords: Fretting fatigue; contact mechanics; normal stress distribution; tangential stress distribution

\section{INTRODUCTION}

Fretting fatigue is a combination of the tribological fretting phenomenon and the failure mechanism fatigue. A schematic overview of fretting fatigue is given in Figure 1, where a specimen and a pad are in contact with each other. The specimen is dynamically loaded with a fatigue force $F_{\text {fat }}$ that governs the fatigue mechanism. The fretting phenomenon is induced by the pad that is normally stressed $\left(F_{N}\right)$ onto the specimen and is tangentially loaded with a dynamic load $Q$, leading to relative displacements in the order of a few micrometer.



Figure 1. Overview of interacting forces $F_{N}, Q \& F_{\text {fat }}$ on fretting fatigue contact between pad and specimen.

An example of practical applications are joints, which are by design and purpose sensitive to fretting fatigue. Connections consist always out of two or more parts that are in contact with each other. Together they sustain a (dynamic) load or are subjected to neighbouring vibrations. It is clear that hereby the conditions are satisfied to obtain fretting fatigue. Joining techniques where fretting fatigue has been encountered are lap-joints [1], dovetail connections [2, 3] and shrink connections [4], see Figure 2.


Figure 2. Overview of some applications where fretting fatigue has been reported.

Compared to plane fatigue conditions, lifetime will be reduced due to fretting fatigue conditions. This is attributed to the stress state that is introduced by the combination of fretting and fatigue loading. The extra stresses due to fretting are concentrated in the contact area and the vicinity of the contact. Not surprisingly, in this area cracks first initiate, then propagate and finally failure takes place. 
To study the influence of the combined fretting and fatigue loading on the lifetime, one should first know the stress state in and nearby the contact area. In literature we find several analytical and numerical techniques to calculate stress states, however a comprehensive approach valid for all loading combinations is unknown to the authors. This paper gives an overview of some analytical formulas available in literature to calculate (normal and tangential) stress distributions, and combines them in a unified procedure.

\section{CONTACT MECHANICS IN FRETTING FATIGUE CONTACTS}

Interaction between two specimens raises stresses in the contact area and the vicinity of the contact area. The interacting forces $\left(F_{N}, Q, F_{F A T}\right)$ introduce a normal stress distribution $p(x)$ and a tangential stress distribution $q(x)$ between the specimens. The purpose of this paper, and developed Matlab script, is to calculate $p(x)$ and $q(x)$ analytically in function of all possible combinations of the normal force $F_{N}$, the tangential force $Q$ and the fatigue force $F_{F A T}$.

The geometry examined in this model is an idealized cylinder-on-flat contact, which is simplified to a 2D plane strain model. This contact type is chosen because it is most frequently used in fretting fatigue research, both numerical and experimental. For example, in finite element simulations this simple geometry is used as a reference to validate the developed numerical models. Cylinder-on-flat contacts are frequently used for coupon scale fretting fatigue experiments. This because the specimens are simple and a reasonable repeatability and controllability of the experiment is realized.

A second boundary condition of the presented model is that both components (specimen and pad) should have the same mechanical properties: Young modulus $E$, and Poisson coefficient $v$. This implies that the Dunders' parameter $\beta$ [5] becomes zero and there is a decoupling between the normal stress distribution $p(x)$ and the tangential stress distribution $q(x)$. A decoupling ensures that the tangential stress distribution $q(x)$ does not affect the normal stress distribution $p(x)$, and vice versa. Therefore, in this paper the calculation for both stress distributions, $p(x)$ and $q(x)$, is tackled in different sub-sections.

\subsection{Normal stress distribution (Hertz)}

When a flat and cylindrical surface are loaded by a normal force $F_{N}$ (Figure $1, Q=0, F_{\text {fat }}=0$ ), the normal stress distribution is given by Hertz' well-known contact theory. Hertz' theory is based on the assumption that both contacting specimens behave as half planes in the vicinity of the contact area, i.e. non-conformal contact. Practically we assume that a contact is non-conformal when $a / R<0,3$ [6], with $a$ the half contact width and $R$ the radius of the cylindrical surface.

A second assumption requires that the contact is incomplete, which means that the contact area cannot be fixed geometrically, for example, dependent on the normal force $F_{N}$ as is the case in Figure 1.

For cylinder-on-flat contacts in fretting fatigue experiments are the boundary conditions for Hertz' theory mostly satisfied. The Hertz' normal pressure distribution $p(x)$ is then given by a parabola: Figure 3 , formula1 [7]. The width of the parabola is $2 a$, where $a$ is given by (2, the maximum normal stress $p_{\max }$ is given by formula 3.

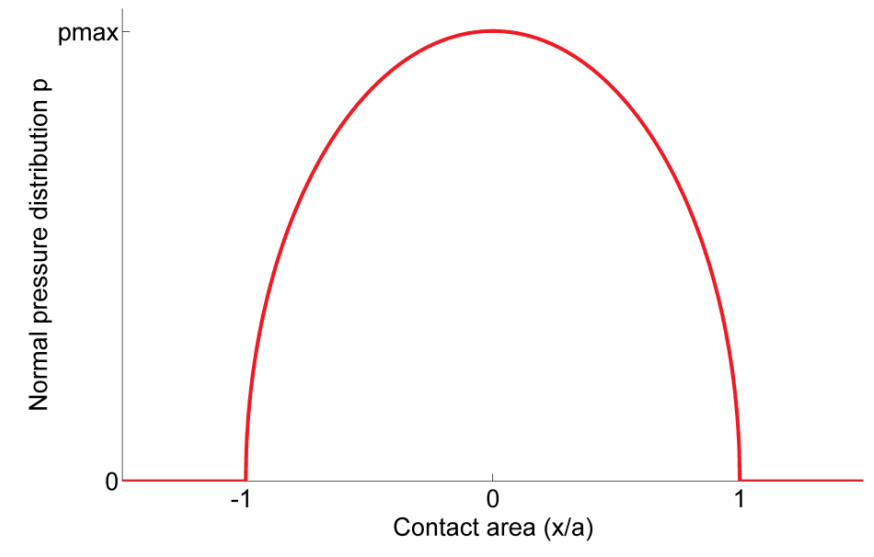

Figure 3. Normal pressure distribution (Hertz) in a line contact.

$$
\mathrm{p}(\mathrm{x})=\mathrm{p}_{\max } \sqrt{1-\left(\frac{x}{a}\right)^{2}}, \quad|x|<a
$$




$$
\begin{gathered}
\mathrm{a}=\sqrt{\frac{8 \cdot \mathrm{R} \cdot \mathrm{F}_{\mathrm{N}} \cdot\left(1-v^{2}\right)}{\pi \cdot \mathrm{E}}} \\
\mathrm{p}_{\text {max }}=\frac{2 \cdot \mathrm{F}_{\mathrm{N}}}{\pi \cdot l \cdot a}
\end{gathered}
$$

The parameters to characterize both materials are the elasticity modulus $E$, and the Poisson coefficient $v$, the length of the contact area $(\perp a)$ is given by $l$.

The implementation of Hertz' theory in Matlab is rather straightforward as shown in Figure 4 as a flow chart. There are three equations: formula (1), (2) and (3) and there is one validation check to verify that the contact is non-conformal instead of conformal. In case of a conformal contact is this theory not able to calculate the normal pressure distribution $p(x)$.

The input parameters of the flowchart are the physical parameters of the problem: $F_{N}, E, v, R$ as well as one computational parameter, xmesh, to discretize the contact area.

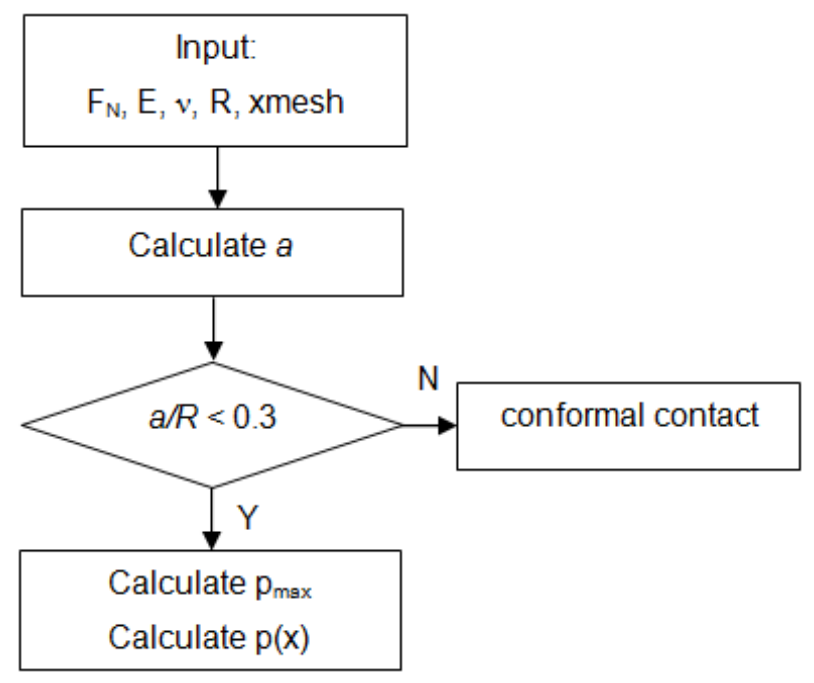

Figure 4. Flow chart to calculate normal stress distribution (Hertz) in Matlab.

\subsection{Tangential stress distribution}

The second stress distribution discussed in this paper is the tangential stress distribution $q(x)$. This stress distribution is more complex than the normal stress distribution $p(x)$ because the tangential stress distribution depends on three forces: $F_{N}, Q$ and $F_{F A T}$ that interact with each other.

There is not one analytical formula which is valid for all combinations of $F_{N}, Q$ and $F_{F A T}$. However, some of the force combinations lead to an analytical solution of the tangential stress distribution $q(x)$. Unfortunately, other combinations of the applied forces cannot be solved analytically, though the tangential stress distribution $q(x)$ can be approximated analytically with an error smaller than one percent [8].

The next paragraph 2.2.1 focuses on contacts with a normal load $F_{N}$ and a tangential load $Q$, the dynamic load $F_{F A T}$ is zero in that case. In the subsequent paragraph 2.2.2 we consider the three forces to interact simultaneously on the specimens. The last part of this section 2.2.3 gives a flowchart to link the discussed theories.

\subsubsection{Normal and tangential loading of a contact}

Consider only a normal force $F_{N}$ between the specimen and the pad in Figure 1. A normal stress distribution appears in the contact area as discussed in section 2.1. In absence of a tangential force $Q$, and because $p(x)$ and $q(x)$ are decoupled, we can conclude that the tangential stress distribution $q(x)$ will be zero over the whole contact area.

If the tangential force $Q$ is bigger than the limiting Coulomb force $\left(\mu F_{N}\right)$, the contact is appointed as global slip. The tangential stress distribution $q(x)$ is similar to the parabolic normal stress distribution $p(x)$, only the amplitude is scaled with the coefficient of friction $\mu$, see Figure 5. 


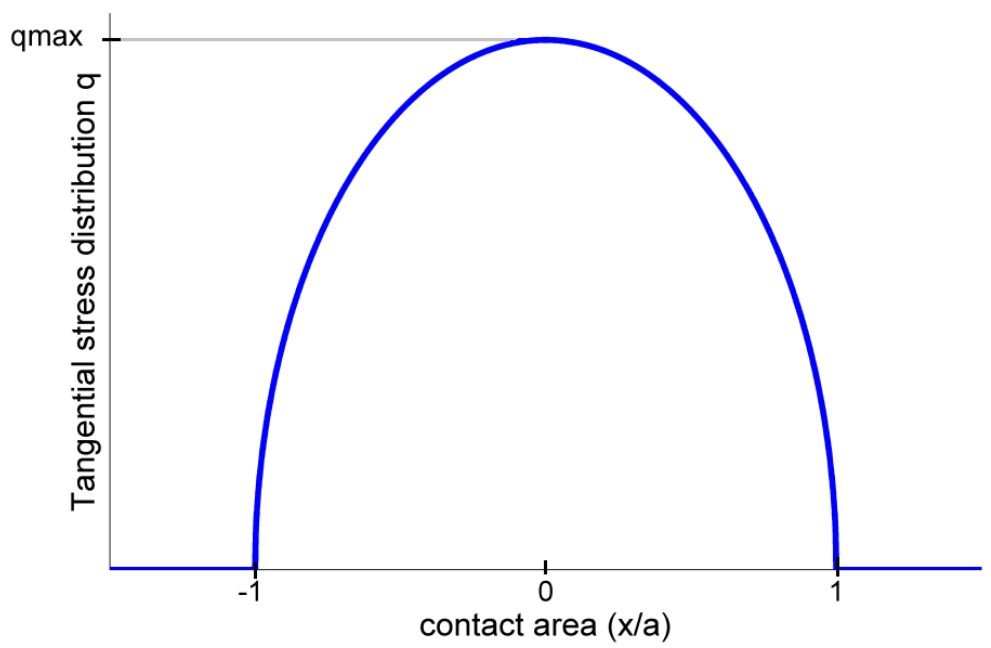

Figure 5. Tangential stress distribution $q$ in a line contact with $F_{N}>0 ; Q>\mu F_{N} 0 ; F_{\text {fat }}=0$.

A third loading case is most interesting with respect to 'pure fretting' conditions. Pure fretting is found when the tangential load $Q$ is smaller than the limiting Coulomb force $\left( \pm \mu F_{N}\right)$ and different from zero. From the perspective of Coulomb, this type of contact is globally sticking. However in the contact area there is locally some small slip between the contacting specimens. The contact area is locally divided in three areas: one area where the specimens are sticking to each other, and two areas with slip between the specimens. This reasoning is first found by Mindlin [9] and Cattaneo [10]. They assumed initially no slip $(\mu \rightarrow \infty)$ in the contact and calculated analytically the tangential stress distribution $q_{1}(x)$,Figure $6 a$. Combining this stress distribution with the limiting stress distribution given by Coulomb $\left(\mu F_{N}\right)$ indicates which parts of the contact area are slipping: $q_{1}(x)>\mu F_{N}$ and which are sticking: $q_{1}(x)<\mu F_{N}$

An analytical value $c$, formula 4 , can be calculated as the border between the central stick zone $(|x|<c)$ and the surrounding slip zone $(c<|x|<a)$. The tangential stress distribution $q(x)$ is calculated as a subtraction of two parabolas, one is the Coulomb parabola $\left(\mu \mathrm{F}_{\mathrm{N}}\right)$ minus a scaled similar parabola, equation 5 .

$$
\begin{gathered}
\frac{c}{a}=\sqrt{1-\left|\frac{Q}{\mu F_{N}}\right|} \\
q(x)=\mu p_{\max } \sqrt{1-\left(\frac{x}{a}\right)^{2}}-\mu p_{\max }\left(\frac{c}{a}\right) \sqrt{1-\left(\frac{x^{\prime}}{c}\right)^{2}} ;|x|<a ;\left|x^{\prime}\right|<c
\end{gathered}
$$
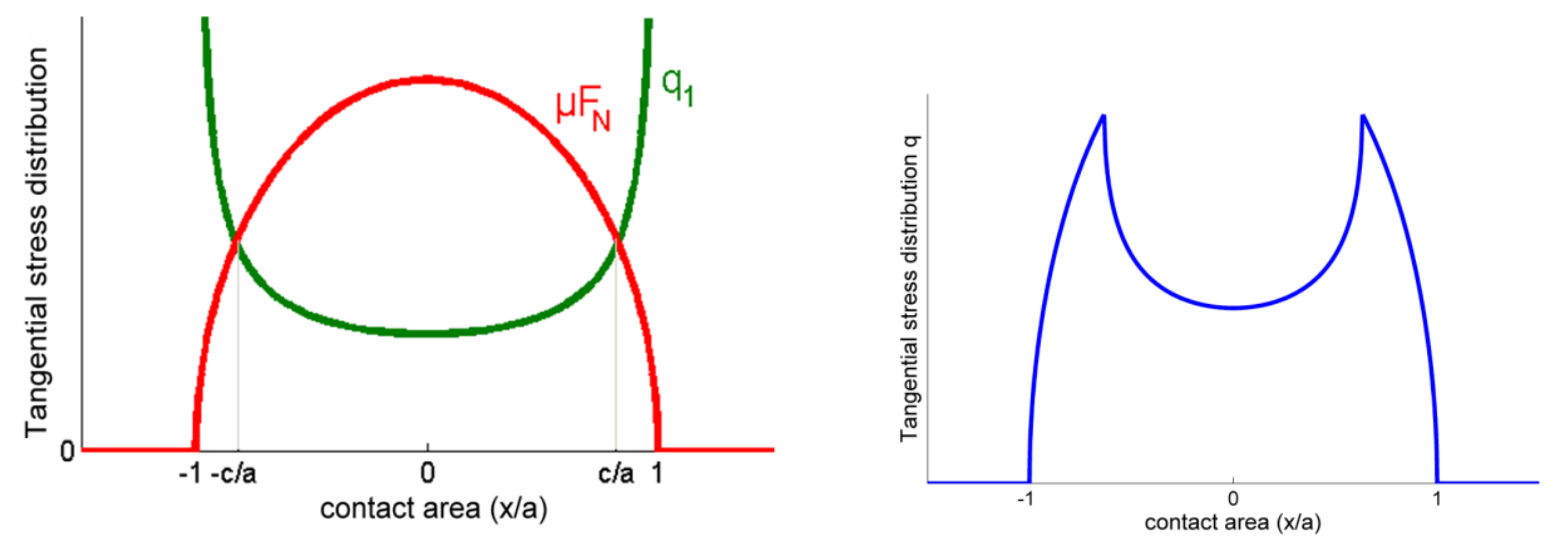

(a) (b)

Figure 6. Tangential stress distribution $q$ in a line contact with $F_{N}>0 ; Q \neq 0 ; F_{\text {fat }}=0$. 


\subsubsection{Combination of normal, tangential, and fatigue loading in a contact}

Addition of a global fatigue load on top of the fretting problem complicates the tangential stress distribution. Most remarkable is that the fatigue load can reverse the direction of the slip within the contact area. When only a normal force $F_{N}$ and a fatigue force $F_{F A T}$ are applied in Figure 1, the tangential stress distribution should be in equilibrium $(Q=0)$. However, physically seen can the tangential stress distribution $q(x)$ not be zero over the whole contact area because the fatigue specimen is axial loaded/stained, where the pad is axial unloaded. This loading mismatch results in a tangential stress distribution $q(x)$ between the two specimens which is point symmetric in $x=0$ (Figure 7). The analytical solution is given by Tur et.al. in [8], a general overview of the calculation method is given on the next page.

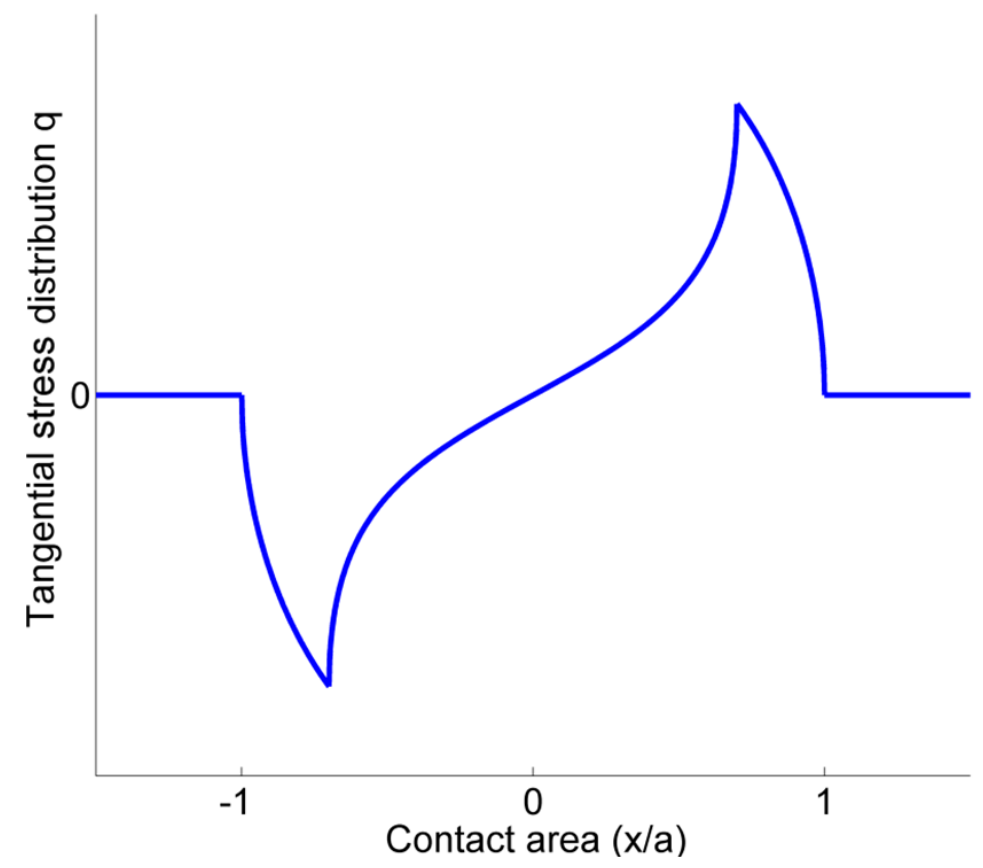

Figure 7. Tangential stress distribution $q$ in a line contact with $F_{N}>0 ; Q=0 ; F_{\text {fat }} \neq 0$.

The last loading condition that is considered in this paper is with all three forces applied in Figure 1 . The combination of tangential force $Q$ and fatigue force $F_{\text {fat }}$ results -as in previous loading cases- in a contact area that is centrally sticking and adjacently slipping. The direction in the slipping areas is governed by $Q$ and $F_{\text {fat }}$, however the local slip in both slipping areas can be in one direction only (cf. $Q \uparrow$ ) or in opposing directions (cf. $F_{\text {fat }} \uparrow$ ). Both cases will be calculated with a different analytical approach, the first tangential stress distribution $q(x)$ for unidirectional slip is analytically given by Hills and Nowell [11]. The second loading case with resulting reverse slip is an approximate calculation given by Tur et. al. [8].

The case of unidirectional slip (cf. $Q \uparrow$ ) in the contact indicates that the tangential stress $q(x)$ is as well unidirectional. The distribution of $q(x)$ was first analytically calculated by Hills and Nowell [11] by a similar reasoning as Mindlin and Cattaneo. Analogous as the latter is the tangential stress distribution $q(x)$ modelled as a perturbation on the limiting Coulomb parabola, formula 6 . The only difference is that the location of the sticking zone is shifted with a distance e compared to the loading case where the fatigue force is absent. The eccentricity $e$ is logically a function of the fatigue force $F_{\text {fat }}$ and can be calculated according to formula 7 .

$$
\begin{gathered}
q(x)=\mu p_{\max } \sqrt{1-\left(\frac{x}{a}\right)^{2}}-\mu p_{\max }\left(\frac{c}{a}\right) \sqrt{1-\left(\frac{x^{\prime}-e}{c}\right)^{2}} ;|x|<a ;\left|x^{\prime}-e\right|<c \\
e=\frac{F_{f a t} a}{4 \mu A p_{\max }}
\end{gathered}
$$


The tangential stress distribution $q(x)$ in this case is show in Figure 8. Note that formula 6 is only valid when $e+c \leq a$, which means that the shifted stick zone should be inside the contact area. However, if the fatigue force $F_{\text {fat }}$ is sufficiently high, the latter boundary condition will be violated and formula 6 is not valid anymore.

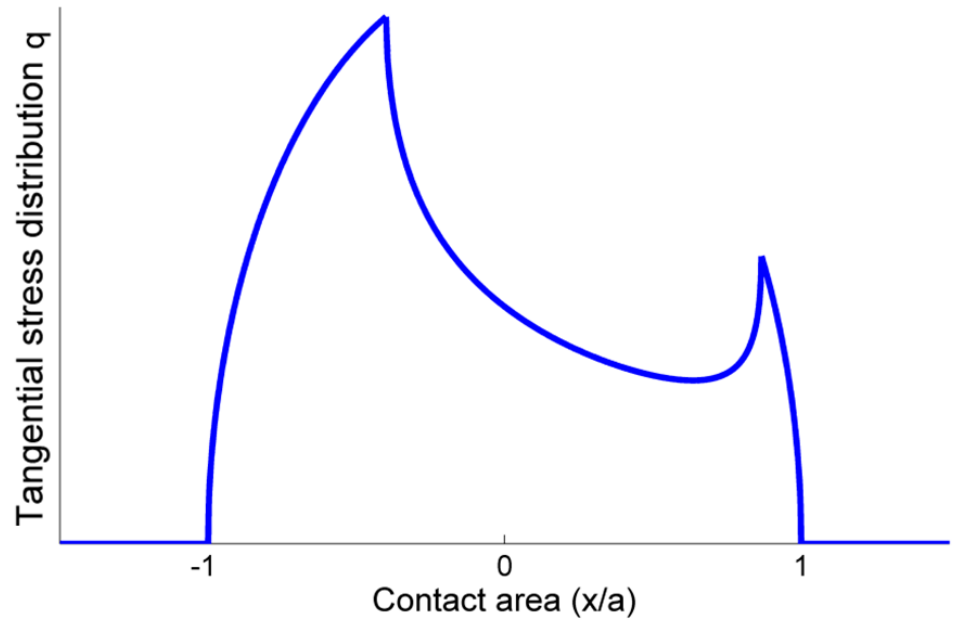

Figure 8. Tangential stress distribution $q$ in a line contact with $F_{N}>0 ; Q \neq 0 ; F_{\text {fat }} \neq 0$.

For the case of high fatigue load and resulting reverse slip it is impossible to calculate the tangential stress distribution $q(x)$ as an analytical closed form solution. Unlike previous solutions we will use an approximated solution to calculate analytically the tangential stress distribution $q(x)$. The approximated solution is given by Tur et.al. in [8], in this paper we will restrict ourselves to a brief overview of the theory.

The contact area is again partitioned in a central stick zone, with surrounding two sliding zones. In the sliding zones we know that $q(x)= \pm \mu p(x)$ (Figure 9a, red dotted line) and that both sliding zones will slip in opposite directions. In the sticking zone of the contact is the tangential stress distribution $q(x)$ approximated by the subtraction of two parabolas (Figure $9 a$, green dashed line), which are parametrically defined by $\left(c^{\prime}, d^{\prime} ; c^{\prime \prime}, d^{\prime \prime} ;\right.$ and two intersections with $\left.\pm \mu p(x)\right)$.

For a given loading case $\left(F_{N}, Q, F_{\text {fat }}\right)$ one can construct a tangential stress distribution based on: $c^{\prime}, d^{\prime}, c^{\prime \prime}, d^{\prime \prime}$ and evaluate how similar this solution is to reality. A least squares fitting algorithm is used to find the best combination of $c^{\prime}, d^{\prime}, c^{\prime \prime}, d^{\prime \prime}$ for a given loading case. For a wide range of loading cases is this optimization performed and four lookup tables are constructed for $c^{\prime}, d^{\prime}, c^{\prime \prime}, d^{\prime \prime}$, based on the parameters $\left(F_{N}, Q\right.$ and $\left.F_{\text {fat }}\right)$.

An analytical method to obtain the tangential stress distribution $q(x)$ for contacts in reverse slip is hereby simplified to the interpolation in four tables and a few summations. This approximation achieves an accuracy better than $1 \%$.

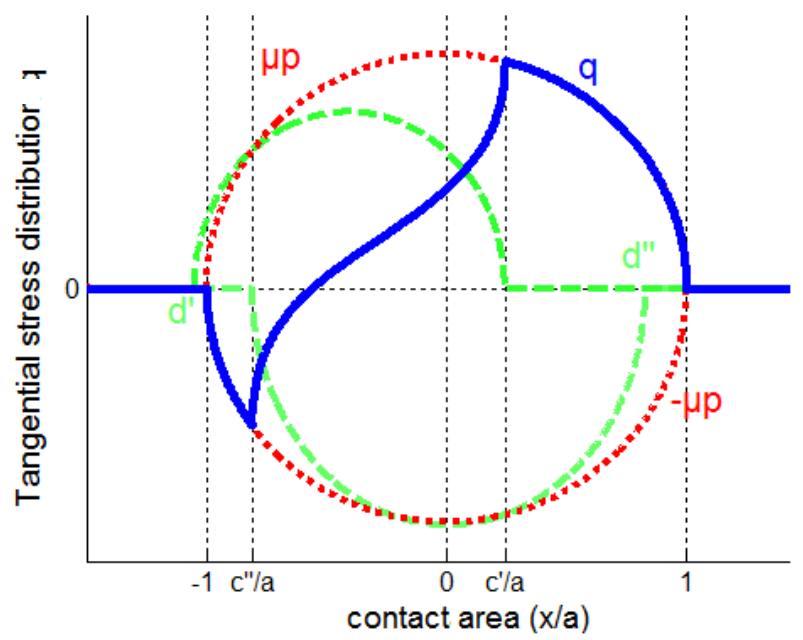

(a)

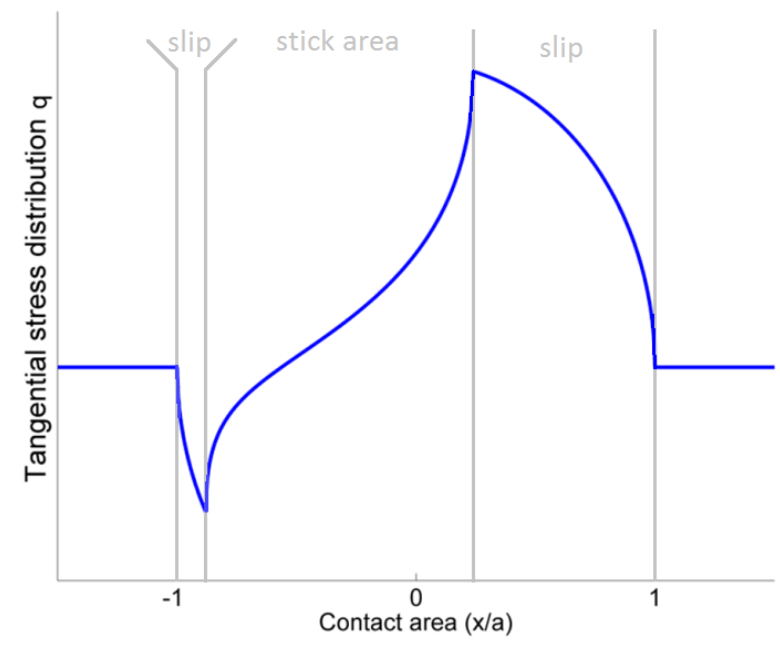

(b)

Figure 9.Tangential stress distribution in a line contact with $F_{N}>0 ; Q \neq 0 ; F_{\text {fat }} \neq 0$.(a) Construction,(b) Result 


\subsubsection{Procedure to calculate the tangential stress distribution $q(x)$ in Matlab}

In previous paragraphs we discussed some analytical solutions to calculate the tangential stress distribution $q(x)$ depending on the applied forces. The link between the theories to obtain a unified approach is given by the flowchart in Figure 10. This flow chart is successfully implemented in a Matlab script at Labo Soete, Ghent University.

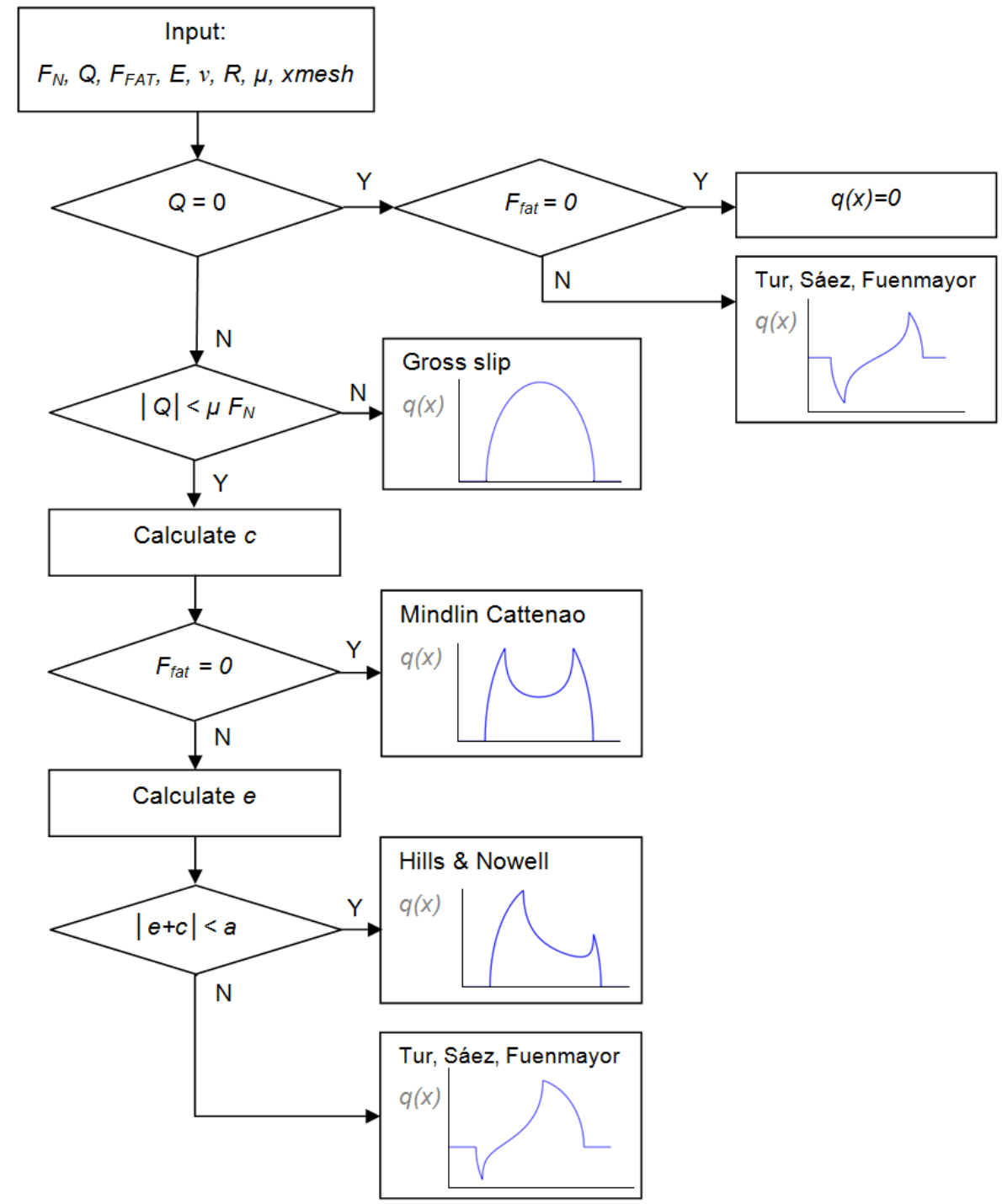

Figure 10. Flowchart to calculate tangential stress $q(x)$.

\section{CONCLUSIONS}

This paper presents a framework to calculate in an analytical way the normal and tangential stress distribution in a fretting fatigue contact as shown in Figure 1. A Matlab script based on this framework is developed at Labo Soete, Ghent University and calculates the normal and tangential stress distribution regardless the combination of applied forces.

Most expressions for the tangential stress distributions are analytically derived and can therefore be used as a reference for other calculation techniques such as finite element calculations. The scripts can later on be extended with crack initiation and crack propagation modules to calculate the lifetime in fretting fatigue conditions. As last, the script can be used to gain basic knowledge about the contact mechanics in fretting fatigue conditions. 


\section{Dunders' parameter}

$\mu \quad$ Coefficient of friction

$v \quad$ Poisson coefficient

a Half width of the contact area

$\mathrm{mm}$

A Cross section $\left(\perp F_{\text {fat }}\right)$ of the fatigue specimen $\mathrm{mm}^{2}$

C Border between symmetrical stick and slip regime $\mathrm{mm}$

c',c" Borders between stick and slip regime $\mathrm{mm}$

d',d" Position of construction parabola (Tur's theory) mm

e Eccentricity of the stick area $\mathrm{mm}$

E Elasticity modulus $\quad \mathrm{MPa}$

$\mathrm{F}_{\mathrm{N}}$ Normal load N N

$F_{\text {fat }}$ Fatigue load N

I Length of the contact area $\mathrm{mm}$

$\mathrm{p} \quad$ Normal stress distribution $\quad \mathrm{MPa}$

q Tangential stress distribution $\quad \mathrm{MPa}$

$\mathrm{q}_{1} \quad$ Tangential stress distribution, assumption of no slip $\mathrm{MPa}$

Q Tangential load N

$\mathrm{R}$ Radius of the cylindrical body $\mathrm{mm}$

$x \quad$ Length co-ordinate in the contact area $\mathrm{mm}$

$x^{\prime} \quad$ Length co-ordinate in the stick area. $\mathrm{mm}$

\section{ACKNOWLEDGEMENTS}

The authors would like to acknowledge the financial support of the IWT, Agency for Innovation by Science and Technology $-n^{\circ}$ SB-091510

\section{REFERENCES}

[1. Chakherlou, T.N., M. Mirzajanzadeh, and J. Vogwell, Experimental and numerical investigations into the effect of an interference fit on the fatigue life of double shear lap joints. Engineering Failure Analysis, 2009. 16(7): p. 2066-2080.

2. Farrahi, G.H., et al., Failure analysis of a gas turbine compressor. Engineering Failure Analysis. 18(1): p. 474-484.

3. Kermanpur, A., et al., Failure analysis of Ti6Al4V gas turbine compressor blades. Engineering Failure Analysis, 2008. 15(8): p. 1052-1064.

4. Alfredsson, B., Fretting fatigue of a shrink-fit pin subjected to rotating bending: Experiments and simulations. International Journal of Fatigue, 2009. 31(10): p. 1559-1570.

5. Dundurs, J. and D.B. Bogy, EDGE-BONDED DISSIMILAR ORTHOGONAL ELASTIC WEDGES UNDER NORMAL AND SHEAR LOADING. Journal of Applied Mechanics, 1969. 36(3): p. 650-\&.

6. Fessler, $\mathrm{H}$. and E. Ollerton, CONTACT STRESSES IN TOROIDS UNDER RADIAL LOADS. British Journal of Applied Physics, 1957. 8(10): p. 387-393.

7. Johnson, K.L., Contact Mechanics. 1985, Cambridge Cambridge University Press.

8. Tur, M., R.C. Saez, and F.J. Fuenmayor, Analytic approach to obtain shear traction in a cylindrical contact with reverse slip. Journal of Strain Analysis for Engineering Design, 2004. 39(6): p. 717727.

9. Mindlin, R.D., Compliance of elastic bodies in contact. Journal of Applied Mechanics, 1949. 16: p. 259-268.

10. Cattaneo, C., Sul Contato di Due Corpo Elastici. Reconditi dell Accademia Nazionale dei Lincei, 1938: p. 342-478.

11. Hills, D.A. and D. Nowell, Mechanics of fretting fatigue. Solid mechanics and its applications, ed. G.M.L. Gladwell. Vol. 30. 1994. 